GEOMETRY IN NONLINEAR CONTROL

AND DIFFERENTIAL INCLUSIONS

BANACH CENTER PUBLICATIONS, VOLUME 32

INSTITUTE OF MATHEMATICS

POLISH ACADEMY OF SCIENCES

WARSZAWA 1995

\title{
WELL-FORMED DYNAMICS UNDER QUASI-STATIC STATE FEEDBACK
}

\author{
J. RUDOLPH \\ Institut für Systemdynamik und Regelungstechnik, Universität Stuttgart \\ D-70550 Stuttgart, Germany \\ E-mail: rudolph@isr.uni-stuttgart.de
}

\begin{abstract}
Well-formed dynamics are a generalization of classical dynamics, to which they are equivalent by a quasi-static state feedback. In case such a dynamics is flat, i.e., equivalent by an endogenous feedback to a linear controllable dynamics, there exists a Brunovský type canonical form with respect to a quasi-static state feedback.
\end{abstract}

1. Introduction. Feedback equivalence and the existence of related canonical forms play a central part in system analysis (see [10, 16, 17, 21, 34]). From a control theoretic viewpoint, equivalence under state feedback is especially interesting. Consequently, there is a lot of nice work, and interesting results are available, in particular for static feedback. However, during the last few years, there has also been considerable interest in dynamic feedback, especially regarding the equivalence to linear controllable systems (see [4, 14, 16, 17, 23, 25, 33] and the references therein).

In the present contribution we are interested in the equivalence of nonlinear dynamics under quasi-static state feedback. This class of feedback, which can be seen as being sort of in-between static and dynamic feedback, has been introduced by Delaleau and Fliess [7] together with an algebraic interpretation of the wellknown structure algorithm. They showed that any classical right invertible system is decouplable via feedback of this type.

In the differential algebraic approach the notion of state is generalized. State representations may depend on time derivatives of the input. In this context, we define the well-formed dynamics which are a generalization of the usual dynamics

1991 Mathematics Subject Classification: 93B10, 93C10, $12 \mathrm{H} 10$.

In this paper the results announced in [28] and [29] are proved and partly corrected.

The author was supported by the Deutsche Forschungsgemeinschaft (DFG).

The paper is in final form and no version of it will be published elsewhere. 
of the type $\dot{x}=f(x, u)$ with full rank jacobian matrix $\frac{\partial f}{\partial u}$ to which they are equivalent by quasi-static feedback of $x$.

Our principal result concerns dynamics that are well-formed and flat, i.e., equivalent to linear controllable dynamics by endogenous feedback [14]. The Brunovský form [3], well-known in the linear theory, is a canonical form for these flat well-formed dynamics under quasi-static feedback of its state. This canonical form can be constructed from any flat output.

2. Mathematical background. We briefly recall the few notions from differential algebra we use. For further details one may see the references [26, 20]. We consider ordinary differential fields of characteristic zero, with the derivation denoted by $\frac{d}{d t}=$ ".". Let $k$ be the differential ground field and $\mathcal{K}$ a differential field containing $k$. We denote as $k(z)$ (resp. $k\langle z\rangle$ ) the fields (resp. differential fields) generated by a finite set $z=\left(z_{1}, \ldots, z_{s}\right)$ of elements of $\mathcal{K}$, and as $\overline{k(z)}$ the algebraic closure of $k(z)$. A finitely generated differential field extension $L / K$ is the datum of differential fields $K$ and $L$ such that $K \subseteq L$, with $L$ (differentially) finitely generated over $K$. The differential field extensions we consider are all finitely generated.

An element $x \in L$ satisfying an algebraic differential equation with coefficients in $K$ is called differentially algebraic over $K$. If every element of $L$ is differentially algebraic over $K$, the extension $L / K$ is called differentially algebraic. Let $\xi=\left(\xi_{1}, \ldots, \xi_{n}\right)$ be a set of elements of $L$. If $\xi$ satisfies an algebraic differential equation with coefficients in $K$, it is called differentially $K$-algebraically dependent. Otherwise, it is called differentially $K$-algebraically independent. Any set of elements of $L$ which is differentially $K$-algebraically independent and maximal with respect to inclusion forms a differential transcendence basis of $L / K$. Any two such bases have the same cardinality, which is called the differential transcendence degree of $L / K$ and denoted as diff $\operatorname{tr} \mathrm{d}^{\circ} L / K$.

A filtration of $L / K$ is a non-decreasing sequence $\mathcal{L}:=\left(\mathcal{L}_{r}\right)_{r \in \mathcal{Z}}$ of (nondifferential) fields $\mathcal{L}_{r}$ such that: $K \subseteq \mathcal{L}_{r} \subseteq L$ and $\mathcal{L}_{r} \subseteq \mathcal{L}_{r+1}$ for all $r \in \mathcal{Z}$, and $\mathcal{L}_{r}=K$ for all $r \in \mathcal{Z}$ small enough. A filtration of $L / K$ is said to be exhaustive if $\cup_{r \in \mathcal{Z}} \mathcal{L}_{r}=L$. We then also say the filtration is exhaustive in $L$. All filtrations we consider are excellent (see [18]). Therefore, they admit so-called Hilbert polynomials $H_{\mathcal{L}}(R)=a R+b \in \mathcal{Q}[R]$ such that for $r$ large enough $\operatorname{tr}^{\circ} \mathcal{L}_{r} / K=H_{\mathcal{L}}(r)$. If, moreover, $\mathcal{L}$ is exhaustive in $L$, the coefficient $a$ in $H_{\mathcal{L}}(R)$ equals the differential transcendence degree of $L / K$ (cf. [18, 32]). Two filtrations $\mathcal{U}$ and $\tilde{\mathcal{U}}$ have bounded (or finite) difference if there exists a non-negative integer $r_{0}$ such that $\mathcal{U}_{r} \subseteq \tilde{\mathcal{U}}_{r+r_{0}}$ and $\tilde{\mathcal{U}}_{r} \subseteq \mathcal{U}_{r+r_{0}}$ for all $r \in \mathcal{Z}[2]$.

For a finitely generated differential field extension $L / k$ introduce the $k$-derivation $d: L \rightarrow \Omega_{L / k}$ with $x \mapsto d x$. Then $d$ satisfies the usual rules of derivation, $\frac{d}{d t} d x=d \dot{x}$, for $x \in L$, and $d a=0$ for $a \in k$. The images $d x$ are called Kähler differentials and $\Omega_{L / k}$ is a (left) $L\left[\frac{d}{d t}\right]$-module called a differential module of Kähler 
differentials. Here $L\left[\frac{d}{d t}\right]$ denotes the ring of polynomials in $\frac{d}{d t}$ with coefficients in $L$. If $L=k\langle z\rangle$, with $z=\left(z_{1}, \ldots, z_{s}\right)$, then $\Omega_{L / k}$ is generated by the set of Kähler differentials $d z=\left(d z_{1}, \ldots, d z_{s}\right)$. An important fact is that $k$-algebraic dependence of a set of elements of $L$ is equivalent to $L$-linear dependence of its differentials. Therefore, for any subfield $k(w)$ of $L$, one has $\operatorname{tr} \mathrm{d}^{\circ} k(w) / k=\operatorname{dim} \operatorname{span}_{L}\{d w\}(\mathrm{cf}$. $[18,19])$.

3. Dynamics. In the differential algebraic approach systems are defined as differential field extensions. The system variables, such as inputs, outputs, and states, are algebraic objects, namely elements of a field. The relations between these objects determine the system equations. We recall the definition which can be found, for example, in [9] or [10].

A system is a (finitely generated) differential field extension $\left({ }^{1}\right) \mathcal{D} / k$. A $d y$ namics, with input $u=\left(u_{1}, \ldots, u_{m}\right)$, is a (finitely generated) differential field extension $\mathcal{D} / k\langle u\rangle$ which is differentially algebraic. The inputs are assumed independent, i.e., they are differential transcendence bases of $\mathcal{D} / k$. A state $x=$ $\left(x_{1}, \ldots, x_{n}\right)$ of a dynamics is a transcendence basis of $\mathcal{D} / k\langle u\rangle$. The (non-differential) transcendence degree of $\mathcal{D} / k\langle u\rangle$ is called the state dimension of the dynamics. It is finite because $\mathcal{D} / k\langle u\rangle$ is differentially algebraic.

Therefore, to such a state $x$ belongs a state representation [9]

$$
A_{i}\left(\dot{x}_{i}, x, u, \dot{u}, \ldots, u^{\left(\alpha_{i}\right)}\right)=0 \quad(i=1, \ldots, n),
$$

where the $A_{i}, i=1, \ldots, n$ are polynomials with coefficients in $k$. Such a state representation (1) is called classical if the orders $\alpha_{i}, i=1, \ldots, n$ are all zero. A state yielding a classical representation is called a classical state. Note that classical states do not necessarily exist for $\mathcal{D} / k\langle u\rangle$ (cf. $[6,8])$.

Two filtrations of $\overline{\mathcal{D}} / k$ are associated with a state $x$ of $\mathcal{D} / k\langle u\rangle$ : a state filtration $\mathcal{X}=\left(\mathcal{X}_{r}\right)_{r \in \mathcal{Z}}$ where

$$
\mathcal{X}_{r}=k \text { for } r \leq-2, \quad \mathcal{X}_{-1}=\overline{k(x)}, \quad \mathcal{X}_{r}=\overline{k\left(x, \dot{x}, \ldots, x^{(r+1)}\right)} \text { for } r \geq 0,
$$

and an input-state filtration $[7] \mathcal{U}=\left(\mathcal{U}_{r}\right)_{r \in \mathcal{Z}}$ where

$$
\mathcal{U}_{r}=k \text { for } r \leq-2, \quad \mathcal{U}_{-1}=\overline{k(x)}, \quad \mathcal{U}_{r}=\overline{k\left(x, u, \dot{u}, \ldots, u^{(r)}\right)} \text { for } r \geq 0 .
$$

Note that $\mathcal{X}_{r}$ (resp. $\mathcal{U}_{r}$ ) contains the derivatives of $x$ (resp. of $u$ ) up to order $r+1$ (resp. $r$ ).

\subsection{Well-formed dynamics}

Definition 3.1. A dynamics $\mathcal{D} / k\langle u\rangle$ is called well-formed if there exists a state $x$, called a well-formed state, of $\mathcal{D} / k\langle u\rangle$ such that the following two conditions are satisfied.

$\left({ }^{1}\right)$ For the sake of simplicity, we identify two systems $\mathcal{D} / k$ and $\tilde{\mathcal{D}} / k$ if there exists a differential $k$-isomorphism between $\mathcal{D}$ and $\tilde{\mathcal{D}}$. 
(i) The state filtration $\mathcal{X}$ is an exhaustive filtration of $\overline{\mathcal{D}} / k$. This is equivalent to $\overline{k\langle x\rangle}=\overline{\mathcal{D}}$.

(ii) The Hilbert polynomial of $\mathcal{X}$ is $H_{\mathcal{X}}(R)=m R+m+n$, where $n$ is the state dimension of $\mathcal{D} / k\langle u\rangle$, i.e., $n=\operatorname{tr} \mathrm{d}^{\circ} \mathcal{D} / k\langle u\rangle$.

Remark. Condition (ii) implies that the Hilbert polynomials of the filtrations $\mathcal{X}$ and $\mathcal{U}$ associated with $x$ are equal.

THEOREM 3.1. Let $\mathcal{D} / k\langle u\rangle$ be a dynamics admitting a classical state $x$ and $\mathcal{X}$ the associated state filtration. Then the following conditions are equivalent:

(i) The filtration $\mathcal{X}$ is an exhaustive filtration of $\overline{\mathcal{D}} / k$.

(ii) The Hilbert polynomial of $\mathcal{X}$ is $H_{\mathcal{X}}(R)=m R+m+n$.

(iii) The jacobian matrix $\frac{\partial A}{\partial u}$ of the state representation (1) belonging to $x$ has full rank $m$.

Proof. One easily verifies that for a classical state representation one has $\operatorname{tr} \mathrm{d}^{\circ} \mathcal{X}_{r} / k=H_{\mathcal{X}}(r)$ for all $r>-2$. One has $\operatorname{tr} \mathrm{d}^{\circ} \mathcal{X}_{-1} / k=H_{\mathcal{X}}(-1)=n$ and $\operatorname{tr} \mathrm{d}^{\circ} \mathcal{X}_{0} / \mathcal{X}_{-1}=\operatorname{rank} \frac{\partial A}{\partial u}$. It follows that $\operatorname{tr} \mathrm{d}^{\circ} \mathcal{X}_{r} / k=H_{\mathcal{X}}(r)=\left(\operatorname{rank} \frac{\partial A}{\partial u}\right)(r+1)+n$ for all $r>-2$. Therefore, (ii) and (iii) are equivalent.

For a classical state representation satisfying the rank condition for $\frac{\partial A}{\partial u}$ one has $\mathcal{U}_{0} \subseteq \mathcal{X}_{0}$, and with this $\mathcal{U}_{r} \subseteq \mathcal{X}_{r}$ for all $r \in \mathcal{Z}$. This implies that the filtration $\mathcal{X}$ is exhaustive in $\overline{\mathcal{D}}$. On the other hand, if rank $\frac{\partial A}{\partial u}<m$, it is obvious that $k(u) \not \subset \mathcal{X}_{r}$ for any $r$. This shows the equivalence of (iii) and (i).

Rem arks. The conditions (i) and (ii) being equivalent, each of those conditions characterizes the well-formed classical states.

A single-input dynamics is well-formed if and only if it admits a classical state representation.

In general, neither of the two conditions of Definition 3.1 implies the other. Take the example $\dot{x}=\dot{u}^{2}$. The Hilbert polynomials of the filtrations $\mathcal{U}$ and $\mathcal{X}$ are $H_{\mathcal{X}}(R)=H_{\mathcal{U}}(R)=R+2$. Nonetheless, $\mathcal{X}$ is not exhaustive in $\overline{\mathcal{D}}$ since it does not contain $u$. As a second example, consider $\dot{x}_{1}=u, \dot{x}_{2}=\dot{u}^{2}$. One easily verifies that $\mathcal{X}$ is exhaustive in $\overline{\mathcal{D}}$ and that the Hilbert polynomial is $H_{\mathcal{X}}(R)=R+1+3$. Observe that, the derivative of $u$ appearing nonlinearly, these two systems do not admit classical state representations $[6,8,15]$. Therefore, by the above remark, they do not admit well-formed states.

4. Quasi-static state feedback. Using the input-state filtrations associated with a state and the input of the dynamics, we may define the quasi-static state feedback relation (cf. [7]).

Definition 4.1. Two dynamics $\mathcal{D} / k\langle u\rangle$ and $\tilde{\mathcal{D}} / k\langle\tilde{u}\rangle$ are said to be related by a quasi-static state feedback if there exist states of $\overline{\mathcal{D}} / k\langle u\rangle$ and $\overline{\tilde{\mathcal{D}}} / k\langle\tilde{u}\rangle$ such that the associated input-state filtrations $\mathcal{U}$ and $\tilde{\mathcal{U}}$ have bounded difference, and $\mathcal{U}_{r}=\tilde{\mathcal{U}}_{r}$ for $r<0$. 
This definition implies the existence of relations between the inputs $u$ and $\tilde{u}$ and a state $x$ of $\overline{\mathcal{D}} / k\langle u\rangle$, which take the form

$$
\begin{aligned}
& \phi_{i}\left(u_{i}, x, \tilde{u}, \dot{\tilde{u}}, \ldots, \tilde{u}^{\left(r_{0}\right)}\right)=0, \\
& \tilde{\phi}_{i}\left(\tilde{u}_{i}, x, u, \dot{u}, \ldots, u^{\left(r_{0}\right)}\right)=0, \quad i=1, \ldots, m .
\end{aligned}
$$

For the state $\tilde{x}$ of $\overline{\tilde{\mathcal{D}}} / k\langle\tilde{u}\rangle$, the equalities $\mathcal{U}_{r}=\tilde{\mathcal{U}}_{r}$ for $r<0$ imply

$$
\psi_{j}\left(x_{j}, \tilde{x}\right)=0, \quad \tilde{\psi}_{j}\left(\tilde{x}_{j}, x\right)=0, \quad j=1, \ldots, n .
$$

This means that the states do not need to be classical, but the state transformations (4) do, i.e., they do not depend on the input or its derivatives. Here, $\phi_{i}, \tilde{\phi}_{i}$, $i=1, \ldots, m$ and $\psi_{j}, \tilde{\psi}_{j}, j=1, \ldots, n$ are all polynomials over $k$.

Given three dynamics such that $\mathcal{D} / k\langle u\rangle$ is related by a quasi-static state feedback to both $\tilde{\mathcal{D}} / k\langle\tilde{u}\rangle$ and $\hat{\mathcal{D}} / k\langle\hat{u}\rangle, \tilde{\mathcal{D}} / k\langle\tilde{u}\rangle$ is not necessarily related by a quasistatic state feedback to $\hat{\mathcal{D}} / k\langle\hat{u}\rangle$. Therefore, the relation defined in Definition 4.1 is not transitive. We get transitivity by specifying the state "fed back" up to classical transformations of the form (4).

Definition 4.2. Two dynamics $\mathcal{D} / k\langle u\rangle$ and $\tilde{\mathcal{D}} / k\langle\tilde{u}\rangle$ are said to be equivalent by quasi-static feedback of a state in $F$ if $F=\overline{k(x)}=\overline{k(\tilde{x})}$, with $x$ (resp. $\tilde{x}$ ) a state of $\mathcal{D} / k\langle u\rangle$ (resp. $\tilde{\mathcal{D}} / k\langle\tilde{u}\rangle)$, and the respective input-state filtrations $\mathcal{U}$ and $\tilde{\mathcal{U}}$ associated with these states have bounded difference.

Of course, if the dynamics are equivalent by quasi-static feedback of a state in $F$, one has $\mathcal{U}_{r}=\tilde{\mathcal{U}}_{r}=F$ for $r<0$. Therefore, if two dynamics are equivalent by quasi-static feedback of a state in $F$, they are related by a quasi-static state feedback, while the opposite is not true in general.

We show that the above indeed defines an equivalence relation on the set of the dynamics for which there exists a state $x$ such that $\overline{k(x)}=F$. Obviously, a dynamics is equivalent to itself by a quasi-static feedback of any of its states. It is also clear that the relation defined in Definition 4.2 is symmetric. Now consider three dynamics such that $\mathcal{D} / k\langle u\rangle$ is equivalent by quasi-static feedback of a state in $F$ to both $\tilde{\mathcal{D}} / k\langle\tilde{u}\rangle$ and $\hat{\mathcal{D}} / k\langle\hat{u}\rangle$. Then the associated filtrations $\mathcal{U}$ and $\tilde{\mathcal{U}}$ have bounded difference, and the same holds for $\mathcal{U}$ and $\hat{\mathcal{U}}$. This means that, for some $r_{0}$, $\mathcal{U}_{r} \subseteq \tilde{\mathcal{U}}_{r+r_{0}}$ and $\tilde{\mathcal{U}}_{r} \subseteq \mathcal{U}_{r+r_{0}}$. Moreover, for some $r_{1}, \mathcal{U}_{r} \subseteq \hat{\mathcal{U}}_{r+r_{1}}$ and $\hat{\mathcal{U}}_{r} \subseteq \mathcal{U}_{r+r_{1}}$. This holds for all $r \in \mathcal{Z}$ and therefore, one has $\hat{\mathcal{U}}_{r} \subseteq \mathcal{U}_{r+r_{1}} \subseteq \tilde{\mathcal{U}}_{r+r_{1}+r_{0}}$ and $\tilde{\mathcal{U}}_{r} \subseteq \mathcal{U}_{r+r_{0}} \subseteq \hat{\mathcal{U}}_{r+r_{1}+r_{0}}$. Hence, the filtrations $\hat{\mathcal{U}}$ and $\tilde{\mathcal{U}}$ have bounded difference, too. Of course, $F=\mathcal{U}_{r}=\tilde{\mathcal{U}}_{r}=\hat{\mathcal{U}}_{r}$ for $r<0$. It follows that the relation defined in Definition 4.2 is also transitive and thus is an equivalence relation.

On the set of dynamics for which classical states exist, we might also introduce equivalence by quasi-static feedback of a classical state, since the classical states are specified up to classical changes of coordinates, i.e., $\overline{k(x)}=\overline{k(\xi)}$ for any classical states $x$ and $\xi$. This mimics the standard situation in control theory where the states are always assumed classical, and where there is no need to distinguish between the states fed back. 
On the contrary, two well-formed states $x$ and $\xi$ of a well-formed dynamics need not be related by a classical transformation: in general, $\overline{k(x)} \neq \overline{k(\xi)}$.

The same considerations apply if one restricts the quasi-static feedback to be a static one, which means that the difference of the filtrations is zero, i.e., they coincide (cf. [7]).

Definition 4.2 implies that the dynamics are equivalent (by endogenous feedback) in the sense of $[14](\overline{\mathcal{D}}=\overline{\tilde{\mathcal{D}}})$. The quasi-static state feedback is a particular type of endogenous feedback [7]. The difference is that in the quasi-static case the state is preserved (up to a classical transformation).

4.1. Brunovský canonical form. A dynamics $\mathcal{D} / k\langle u\rangle$ is flat if there exists a differential transcendence basis $y=\left(y_{1}, \ldots, y_{m}\right)$ of $\overline{\mathcal{D}} / k$, called a flat (or linearizing) output, such that $\overline{k\langle y\rangle}=\overline{\mathcal{D}}$. Such a dynamics is equivalent (by endogenous feedback) to a linear controllable one (cf. [14]).

If the dynamics $\mathcal{D} / k\langle u\rangle$ is flat, the corresponding module of Kähler differentials $\Omega_{\overline{\mathcal{D}} / k}$ is free, and for any flat output $y$ the set $d y=\left(d y_{1}, \ldots, d y_{m}\right)$ is a basis of $\Omega_{\overline{\mathcal{D}} / k}$. The module $\Omega_{\overline{\mathcal{D}} / k}$ is the time-varying linearization of $\overline{\mathcal{D}} / k$. The set of differentials $d u=\left(d u_{1}, \ldots, d u_{m}\right)$ is the input of the associated linear dynamics (cf. e.g. [30]).

The well-known Brunovský form [3] $v_{i}=\omega_{i}^{\left(\kappa_{i}\right)}, i=1, \ldots, m$, with $\kappa_{i}>0$ for $i=1, \ldots, m$, is a canonical form for static state feedback equivalence for the flat (i.e., controllable [14]) linear time-varying dynamics with $m$ inputs. It obviously represents a well-formed dynamics. A formal module theoretic version of this result, which is adapted to our framework, can be found in $[12,13]$. We will now derive an analogous result for nonlinear flat dynamics.

Let there be given a flat dynamics $\mathcal{D} / k\langle u\rangle$. Then $\Omega_{\overline{\mathcal{D}} / k}$ is a free $\overline{\mathcal{D}}\left[\frac{d}{d t}\right]$-module. This implies that for any basis $\bar{\omega}$ of $\Omega_{\overline{\mathcal{D}} / k}$ there exist relations $d u_{i}=\sum_{j=1}^{m} a_{i, j} \bar{\omega}_{j}$, $i=1, \ldots, m$, with the coefficients $a_{i, j} \in \overline{\mathcal{D}}\left[\frac{d}{d t}\right]$. Then, with $\omega=\left(\omega_{1}, \ldots, \omega_{m}\right)$ another basis of $\Omega_{\overline{\mathcal{D}} / k}$, these relations can also be represented by

$$
\mu_{i}=c_{i} \omega_{i}, \quad i=1, \ldots, m,
$$

with $\mu=\left(\mu_{1}, \ldots, \mu_{m}\right)$ a basis of the $\overline{\mathcal{D}}\left[\frac{d}{d t}\right]$-module $[d u]$ generated by $d u=$ $\left(d u_{1}, \ldots, d u_{m}\right)$, and $c_{i} \in \overline{\mathcal{D}}\left[\frac{d}{d t}\right]$. This corresponds to a diagonalization of the matrix $\left(a_{i, j}\right)$ by multiplication on the left and on the right with unimodular matrices over $\overline{\mathcal{D}}\left[\frac{d}{d t}\right]$, which is always possible [5]. The coefficients $c_{i}, i=1, \ldots, m$, are polynomials in $\frac{d}{d t}$. For $i=1, \ldots, m$, denote the degree of $c_{i}$ as $\kappa_{i}$. We call $\kappa_{i}$, $i=1, \ldots, m$ the generalized controllability indices $\left({ }^{2}\right)$ of $\mathcal{D} / k\langle u\rangle$. Up to renumbering, these indices are independent of the choice of $\omega$. This is a consequence of the next lemma.

$\left({ }^{2}\right)$ This characterization is different from the one announced in [28] and [29] which, contrarily to what is claimed there, is not independent of the choice of the flat output. 
Lemma 4.1. Let $\mathcal{D} / k\langle u\rangle$ be a flat dynamics and let $\kappa_{1}, \ldots, \kappa_{m}$ be the degrees of the polynomials $c_{i}, i=1, \ldots, m$ in (5) belonging to the basis $\omega$ of $\Omega_{\overline{\mathcal{D}} / k}$, as defined above. Then, up to renumbering the components of $y$, for any flat output $y$ of $\mathcal{D} / k\langle u\rangle$, the set $x=\left(y_{1}, \ldots, y_{1}^{\left(\kappa_{1}-1\right)}, y_{2}, \ldots, y_{m}^{\left(\kappa_{m}-1\right)}\right)$ is a state of $\overline{\mathcal{D}} / k\langle u\rangle$.

Definition 4.3. The states $\left({ }^{3}\right) x=\left(y_{1}, \ldots, y_{1}^{\left(\kappa_{1}-1\right)}, y_{2}, \ldots, y_{m}^{\left(\kappa_{m}-1\right)}\right)$ defined in the above lemma are called the Brunovsky states of the flat dynamics $\mathcal{D} / k\langle u\rangle$.

One observes that the Brunovský states are unique up to the choice of the flat output $y$. Changing the flat output corresponds to a differential $k$-automorphism of $\overline{\mathcal{D}}$.

Proof. Let $\omega$ be the basis of $\Omega_{\overline{\mathcal{D}} / k}$ yielding the representation (5). Denote as $w_{i}^{i}$ the canonical image of $\omega_{i}$ in $\Omega_{\overline{\mathcal{D}} / k} /\left(\oplus_{j=1, j \neq i}^{m}\left[\omega_{j}\right] \oplus\left[c_{i} \omega_{i}\right]\right)$, and denote the $\overline{\mathcal{D}}\left[\frac{d}{d t}\right]$-module generated by $w_{i}^{i}$ as $\left[w_{i}^{i}\right]$. Then one has an isomorphism between the $\overline{\mathcal{D}}\left[\frac{d}{d t}\right]$-modules $\Omega_{\overline{\mathcal{D}} / k} /[d u]$ and $\oplus_{i=1}^{m}\left[w_{i}^{i}\right]$. Each $\left[w_{i}^{i}\right]$ is a cyclic torsion module the dimension of which, when considered as a $\overline{\mathcal{D}}$-vector space, is equal to the degree of the corresponding coefficient $c_{i}$. For any basis $\bar{\omega}$ of $\Omega_{\overline{\mathcal{D}} / k}$, denote as $\bar{w}_{i}$ the canonical image of $\bar{\omega}_{i}$ in the $\overline{\mathcal{D}}$-vector space $\Omega_{\overline{\mathcal{D}} / k} /[d u]$. It can be shown that, up to renumbering its components, the set $\xi=\left(\bar{w}_{1}, \dot{\bar{w}}_{1}, \ldots, \bar{w}_{1}^{\left(\kappa_{1}-1\right)}, \bar{w}_{2}, \ldots, \bar{w}_{m}^{\left(\kappa_{m}-1\right)}\right)$ is a basis of the $\overline{\mathcal{D}}$-vector space $\Omega_{\overline{\mathcal{D}} / k} /[d u]$, as follows. The $\overline{\mathcal{D}}$-vector space spanned by $\xi$ is isomorphic to both $\Omega_{\overline{\mathcal{D}} / k} /[d u]$ and $\oplus_{i=1}^{m}\left[w_{i}^{i}\right]$. For, when considered as $\overline{\mathcal{D}}$-vector spaces, each $\left[w_{i}^{i}\right]$ is isomorphic to a subspace of $\left[\bar{w}_{i}\right]$, up to renumbering. This can be seen by inspecting the relations between two bases. By renumbering, one gets an expression of the form $\bar{w}_{i}^{i}=a_{i} w_{i}^{i}$, with $0 \neq a_{i} \in \overline{\mathcal{D}}\left[\frac{d}{d t}\right]$, for the image $\bar{w}_{i}^{i}$ of $\bar{\omega}_{i}$ in $\left[w_{i}^{i}\right]$. From this, by derivation, one sees that the dimension of the $\overline{\mathcal{D}}$-vector space $\left[\bar{w}_{i}^{i}\right]$ is $\kappa_{i}$. Consequently, there is a $\kappa_{i}$-dimensional subspace of the $\overline{\mathcal{D}}$-vector space $\left[\bar{w}_{i}\right]$ isomorphic to $\left[w_{i}^{i}\right]$. Finally, a non-trivial $\overline{\mathcal{D}}$-linear dependence relation between the elements of $\xi$ would result in the same relation between the elements of the bases of the $\left[w_{i}^{i}\right]$. This being excluded by the direct sum property, $\xi$ actually is a basis of the $\overline{\mathcal{D}}$-vector space $\Omega_{\overline{\mathcal{D}} / k} /[d u]$.

The differential of any flat output $y$ of $\mathcal{D} / k\langle u\rangle$ is a basis of the corresponding module $\Omega_{\overline{\mathcal{D}} / k}$. Therefore, the family of canonical images in $\Omega_{\overline{\mathcal{D}} / k} /[d u]$ of $d y_{1}, \dot{d} y_{1}, \ldots, d y_{1}^{\left(\kappa_{1}-1\right)}, d y_{2}, \ldots, d y_{m}^{\left(\kappa_{m}-1\right)}$ is $\overline{\mathcal{D}}$-linearly independent. The proof is thus concluded by observing that this implies that $\left(y_{1}, \dot{y}_{1}, \ldots, y_{1}^{\left(\kappa_{1}-1\right)}, y_{2}, \ldots\right.$ $\left.\ldots, y_{m}^{\left(\kappa_{m}-1\right)}\right)$ is a $k\langle u\rangle$-algebraically independent family.

Remark. In the above proof, the basis $\omega$ of $\Omega_{\overline{\mathcal{D}} / k}$ does not have to be composed of differentials of elements of $\overline{\mathcal{D}}$. This corresponds to non-exact one-

$\left({ }^{3}\right)$ Strictly spoken, a Brunovský state is not necessarily a state of $\mathcal{D} / k\langle u\rangle$, but only of $\overline{\mathcal{D}} / k\langle u\rangle$. 
forms in the differential geometric approach (cf. [25]). Nonetheless, for each flat output there is a corresponding basis of $\Omega_{\overline{\mathcal{D}} / k}$.

LEMma 4.2. Let $\mathcal{D} / k\langle u\rangle$ be a flat dynamics. Then the Brunovsky states of $\mathcal{D} / k\langle u\rangle$ are well-formed if and only if $\kappa_{i}>0, i=1, \ldots, m$.

Proof. The necessity follows from the fact that a well-formed state must contain a flat output in order that the corresponding state filtration can be exhaustive. Of course, if $\kappa_{i}>0, i=1, \ldots, m$, the Brunovský state $x$ constructed with the flat output $y$ contains $y$. Therefore, the associated state-filtration $\mathcal{X}$ is exhaustive in $\overline{\mathcal{D}}$. This implies that $H_{\mathcal{X}}(R)=m R+b$. Moreover, $x$ is constructed in such a way that $\operatorname{tr}^{\circ} k(x, \dot{x}) / k(x)=m$. Therefore, $\operatorname{tr} \mathrm{d}^{\circ} \mathcal{X}_{r} / \mathcal{X}_{r-1}=m$ for all $r \geq 0$, and it follows that $H_{\mathcal{X}}(R)=m(R+1)+\operatorname{tr}^{\circ} \mathcal{X}_{-1} / \mathcal{X}_{-2}=m(R+1)+n$.

Traditionally, states are understood to be classical states, and, as mentioned above, one need not distinguish the states in the definition of feedback equivalence in this case. In our general context this is different. However, it is obvious that the generalized controllability indices form a set of invariants for the equivalence by quasi-static feedback of a state in $F$ on the set of the flat dynamics if we restrict the field $F$ to be generated over $k$ by a Brunovský state.

Definition 4.4. Two flat dynamics $\mathcal{D} / k\langle u\rangle$ and $\tilde{\mathcal{D}} / k\langle\tilde{u}\rangle$ are said to be equivalent by quasi-static feedback of a Brunovský state if for some Brunovský states $x$ and $\tilde{x}$ of these dynamics one has $\overline{k(x)}=\overline{k(\tilde{x})}=: F$, and $\mathcal{D} / k\langle u\rangle$ and $\tilde{\mathcal{D}} / k\langle\tilde{u}\rangle$ are equivalent by quasi-static feedback of a state in $F$.

The above condition is equivalent to the condition that the input-state filtrations of $\mathcal{D} / k\langle u\rangle$ and $\tilde{\mathcal{D}} / k\langle\tilde{u}\rangle$ associated with respective Brunovský states $x$ and $\tilde{x}$ have bounded difference and $\overline{k(x)}=\overline{k(\tilde{x})}$. It is easy to see that the relation defined in Definition 4.4 is an equivalence relation on the set of flat dynamics, by identifying appropriate flat outputs via a differential $k$-isomorphism between the corresponding fields.

Definition 4.5. The dynamics $k\langle y, v\rangle / k\langle v\rangle$ such that $v_{i}=y_{i}^{\left(\kappa_{i}\right)}$, with $\kappa_{i}>0$, $i=1, \ldots, m$, is called the Brunovský form of a flat well-formed dynamics.

THEOREM 4.1. The Brunovsky form is a canonical form for the equivalence by quasi-static feedback of a Brunovsky state on the set of the well-formed flat dynamics with well-formed Brunovsky states.

In other words, to every flat well-formed dynamics $\mathcal{D} / k\langle u\rangle$ with well-formed Brunovský states belongs a unique dynamics $k\langle y, v\rangle / k\langle v\rangle$, with $\overline{\mathcal{D}}=\overline{k\langle y, v\rangle}$, and a unique state representation in Brunovský form. This canonical form can be constructed from any flat output. The corresponding dynamics $\overline{\mathcal{D}} / k\langle v\rangle$ is equivalent to $\mathcal{D} / k\langle u\rangle$ by a quasi-static feedback of a state in $\overline{k(x)}$. Here $x$ is the Brunovský state of $\mathcal{D} / k\langle u\rangle$, which, in general, need not be a classical state of $\overline{\mathcal{D}} / k\langle u\rangle$. 
Pr o of. Let $x$ be a well-formed Brunovský state of a flat well-formed dynamics $\mathcal{D} / k\langle u\rangle$. Furthermore, let $v$ be the input of the corresponding Brunovský form, i.e., $v_{i}=y_{i}^{\left(\kappa_{i}\right)}, i=1, \ldots, m$, and $\mathcal{V}$ the input-state filtration of $\overline{\mathcal{D}} / k\langle v\rangle$ associated with $x$. We show that $\mathcal{D} / k\langle u\rangle$ and $\overline{\mathcal{D}} / k\langle v\rangle$ are related by a quasi-static state feedback. The filtrations $\mathcal{X}$ and $\mathcal{V}$ coincide, because all but $m$ components of $\dot{x}$ are elements of $x$, and the others are equal to the components of $v$. The filtrations $\mathcal{V}$ and $\mathcal{U}$ have bounded difference, $r_{0}$ say. This can be seen as follows. For $r_{0}$ large enough, on one hand, the definition of a state implies $\dot{x} \subset \mathcal{U}_{r_{0}}$, wherefrom with $\mathcal{X}_{0}=\mathcal{V}_{0}$ it follows $\mathcal{V}_{0} \subseteq \mathcal{U}_{r_{0}}$, and from this $\mathcal{V}_{r} \subseteq \mathcal{U}_{r+r_{0}}$ for all $r>0$. On the other hand, by Lemma $4.2, x$ contains a flat output and thus $\mathcal{X}$ is exhaustive in $\overline{\mathcal{D}}$. Hence, $\mathcal{U}_{0} \subseteq \mathcal{V}_{r_{0}}$, and $\mathcal{U}_{r} \subseteq \mathcal{V}_{r+r_{0}}$ for all $r>0$.

The state $x$ is a Brunovský state of $\overline{\mathcal{D}} / k\langle v\rangle$. By construction, it is also a (not necessarily classical) Brunovský state of $\overline{\mathcal{D}} / k\langle u\rangle$. The field $F=\overline{k(x)}$ has been kept invariant. We conclude that $\mathcal{D} / k\langle u\rangle$ and $\overline{\mathcal{D}} / k\langle v\rangle$ are related, and hence equivalent, by quasi-static feedback of a Brunovský state. Finally, the uniqueness of the Brunovský form follows from Lemma 4.1.

Recall that all flat dynamics are equivalent to a Brunovský form via endogenous feedback, but this form is not unique in that case [14]. In our case it is unique because of the restriction that $\overline{k(x)}$ is invariant and must contain Brunovsky states of both $\mathcal{D} / k\langle u\rangle$ and $\tilde{\mathcal{D}} / k\langle\tilde{u}\rangle$, and these correspond to a unique Brunovský form. In contrast, a dynamics may generally be related by a quasi-static state feedback to different Brunovský forms (cf. the remarks following Definition 4.1). This can easily be seen on the proof of Theorem 4.1 , by letting the set $x$ in the proof be composed of $y$ itself and any derivatives of the components of $y$ such that $x$ is a state of $\overline{\mathcal{D}} / k\langle u\rangle$. We formulate this as a corollary.

COROLlary 4.1. Let $\mathcal{D} / k\langle u\rangle$ be a flat dynamics such that the flat output $y$ is a differentially $k\langle u\rangle$-algebraically independent family. Then, if, for some $l_{1}, l_{2}, \ldots, l_{m}>0$, the set $x=\left(y_{1}, \ldots, y_{1}^{\left(l_{1}-1\right)}, y_{2}, \ldots, y_{m}^{\left(l_{m}-1\right)}\right)$ is a state of $\overline{\mathcal{D}} / k\langle u\rangle$, the dynamics $\mathcal{D} / k\langle u\rangle$ is related by a quasi-static state feedback to a Brunovský form with (generalized) controllability indices $l_{1}, l_{2}, \ldots, l_{m}$.

We may also characterize the whole set of the dynamics being equivalent to a Brunovský form by quasi-static feedback of a Brunovský state. Necessarily, they are flat, since they are equivalent to a linear controllable dynamics [14]. They are equivalent by a quasi-static feedback of $x$ to a dynamics with a classical representation belonging to $x$, namely the Brunovský form. Therefore, it follows from Theorem 4.2 below that they are also well-formed. As a consequence, the set of the well-formed flat dynamics with $\kappa_{i} \neq 0, i=1, \ldots, m$ is the maximal set (w.r.t. set inclusion) admitting the Brunovský canonical form for the equivalence by quasi-static feedback of a Brunovský state.

R e m a r k. It is not difficult to observe that if a Brunovský state is a classical state, it leads to static state feedback equivalence to the Brunovský form. (We 
did not define static feedback equivalence here, cf. [7].)

R e m ark. In the linear case, time-varying or not, the result in Theorem 4.1 shows that there is a Brunovský canonical form for the equivalence by quasi-static feedback of a Brunovský state for the well-formed controllable linear systems. This canonical form can be obtained from any basis of the system module (cf. [31]). On the contrary, for the classical static state feedback equivalence this canonical form is obtained from particular bases (see $[12,13]$ ).

4.2. Well-formed dynamics and classical representations. The following theorem shows the role of well-formed dynamics for classical state representations.

TheOrem 4.2. A dynamics $\mathcal{D} / k\langle u\rangle$ is equivalent by quasi-static feedback of a state in $\overline{k(x)}$ to a dynamics $\tilde{\mathcal{D}} / k\langle\tilde{u}\rangle$ admitting a classical state representation $A_{i}\left(\dot{x}_{i}, x, \tilde{u}\right)=0, i=1, \ldots, n$ with $\frac{\partial A}{\partial \tilde{u}}$ of full rank $m$ if and only if $\mathcal{D} / k\langle u\rangle$ is well-formed. Then the state $x$ is a well-formed state of $\overline{\mathcal{D}} / k\langle u\rangle$, too.

P r o of. Let $x$ be a well-formed state of $\mathcal{D} / k\langle u\rangle$. The Hilbert polynomial of $\mathcal{X}$ is $H_{\mathcal{X}}(R)=m(R+1)+n$, where $n$ is the state dimension of $\mathcal{D} / k\langle u\rangle$. One easily verifies that $\operatorname{tr} \mathrm{d}^{\circ} \mathcal{X}_{0} / \mathcal{X}_{-1}<m$ would yield $H_{\mathcal{X}}(R)<m(R+1)+n$. Therefore, $\operatorname{tr} \mathrm{d}^{\circ} \mathcal{X}_{0} / \mathcal{X}_{-1}=m$, allowing to introduce a new input $\tilde{u}$ as a transcendence basis of $\mathcal{X}_{0} / \mathcal{X}_{-1}$. Then the filtrations $\mathcal{X}$ and $\tilde{\mathcal{U}}$ of $\tilde{\mathcal{D}} / k\langle\tilde{u}\rangle$ associated with $x$ coincide. This implies that the state representation of $\tilde{\mathcal{D}} / k\langle\tilde{u}\rangle$ belonging to $x$ is classical (one may put $\tilde{\mathcal{D}}=\overline{\mathcal{D}}$ ). Now we show that $\mathcal{U}$ and $\tilde{\mathcal{U}}$ have bounded difference. Let $r_{0}$ be sufficiently large. Then, $\mathcal{X}=\tilde{\mathcal{U}}$ being exhaustive in $\overline{\mathcal{D}}$, the inclusion $\mathcal{U}_{0} \subseteq \tilde{\mathcal{U}}_{r_{0}}$ follows. The other way round is a consequence of the definition of a state: There always exists a non-negative $r_{0}$ such that $\dot{x} \subset \mathcal{U}_{r_{0}}$, and with this $\mathcal{X}_{0}=\tilde{\mathcal{U}}_{0} \subseteq \mathcal{U}_{r_{0}}$. Moreover, the construction of the filtrations implies that $\mathcal{U}_{s} \subseteq \tilde{\mathcal{U}}_{r_{0}+s}$ and $\tilde{\mathcal{U}}_{s} \subseteq \mathcal{U}_{r_{0}+s}$ for positive $s$. Finally, by introducing the new input $\tilde{u}$ as a transcendence basis of $\mathcal{X}_{0} / \mathcal{X}_{-1}$, the field $\mathcal{X}_{-1}$, and with this all $\mathcal{X}_{r}, r<0$, are kept invariant.

Now consider a classical state representation with state $x$ of $\tilde{\mathcal{D}} / k\langle\tilde{u}\rangle$ which satisfies the rank condition for $\frac{\partial A}{\partial \tilde{u}}$. One has $\mathcal{X}_{r}=\tilde{\mathcal{U}}_{r}$ for all $r$, whence $H_{\mathcal{X}}(R)=$ $H_{\tilde{\mathcal{U}}}(R)=m R+m+n$. If $\mathcal{D} / k\langle u\rangle$ and $\tilde{\mathcal{D}} / k\langle\tilde{u}\rangle$ are equivalent by quasi-static feedback of a state in $\mathcal{X}_{-1}$, one has $\mathcal{U}_{0} \subseteq \tilde{\mathcal{U}}_{r_{0}}=\mathcal{X}_{r_{0}}$, for a sufficiently large non-negative integer $r_{0}$. Consequently, $\mathcal{U}_{s} \subseteq \mathcal{X}_{r_{0}+s}$ for all $s>0$, and thus $\mathcal{X}$ is exhaustive in $\overline{\mathcal{D}}$. We conclude that $\mathcal{D} / k\langle u\rangle$ is well-formed and that $x$ is a well-formed state of $\overline{\mathcal{D}} / k\langle u\rangle$.

R e mark. This result can be interpreted as a solution to a generalization of the problem of equivalence to classical representations under non-classical state coordinate changes, as considered and solved in $[15,6,8]$. For, if there is a classical state of $\mathcal{D} / k\langle u\rangle$, we may choose this state in the theorem, and get equivalence by quasi-static feedback of a state in $\overline{k(x)}$ to a dynamics $\tilde{\mathcal{D}} / k\langle\tilde{u}\rangle$ by defining $\tilde{u}=u$. 
5. Concluding remarks. Using well-formed representations, an extension of recent results on feedback decoupling and inversion [7] to generalized state representations involving time derivatives of the input variables should be possible.

As a variation of the classical state realization problem, one might hope to get a set of simple conditions for the existence of well-formed states. Lemma 4.2 provides such a condition for the flat dynamics, which are important in applications (see e.g. [14, 27, 28, 29]).

\section{References}

[1] R. Aris, Introduction to the Analysis of Chemical Reactors, Prentice-Hall, Englewood Cliffs, 1965.

[2] M. F. Atiyah and I. G. Macdonald, Introduction to Commutative Algebra, AddisonWesley, Reading, 1969.

[3] P. Brunovský, A classification of linear controllable systems, Kybernetika 6 (1970), 173-187.

[4] B. Charlet, J. Lévine and R. Marino, Sufficient conditions for dynamic state feedback linearization, SIAM J. Control Optim. 29 (1991), 38-57.

[5] P. M. Cohn, Free Rings and their Relations, 2nd edition, Academic Press, London, 1985.

[6] E. Delaleau, Lowering orders of input derivatives in generalized state representations of nonlinear systems, in: Proc. IFAC-Symposium NOLCOS'92, Bordeaux, M. Fliess (ed.), 1992, 209-213.

[7] E. Delaleau et M. Fliess, Algorithme de structure, filtrations et découplage, C. R. Acad. Sci. Paris Sér. I 315 (1992), 101-106.

[8] E. Delaleau and W. Respondek, Lowering the orders of derivatives of controls in generalized state space systems, J. Math. Systems Estim. Control, to appear.

[9] M. Fliess, Automatique et corps différentiels, Forum Math. 1 (1989), 227-238.

[10] -, Generalized controller canonical forms for linear and nonlinear dynamics, IEEE Trans. Automat. Control 35 (1990), 994-1001.

[11] -, Some basic structural properties of generalized linear systems, Systems Control Lett. 15 (1990), 391-396.

[12] - Some remarks on a new characterization of linear controllability, in: Prepr. 2nd IFAC Workshop "System Structure and Control", Prague, Sept. 1992, 8-11.

[13] - Some remarks on the Brunovský canonical form, Kybernetika 29 (1993), 417-422.

[14] M. Fliess, J. Lévine, P. Martin et P. Rouchon, Sur les systèmes non linéaires différentiellement plats, C. R. Acad. Sci. Paris Sér. I 315 (1992), 619-624.

[15] S. T. Glad, Nonlinear state space and input output descriptions using differential polynomials, in: New Trends in Nonlinear Control Theory, J. Descusse, M. Fliess, A. Isidori and D. Leborgne (eds.), Lecture Notes Control Inform. Sci. 122, Springer, 1988, 182-189.

[16] A. Isidori, Nonlinear Control Systems: An Introduction, Springer, New York, 1989.

[17] B. Jakubczyk, Dynamic feedback equivalence of nonlinear control systems, preprint.

[18] J. Johnson, Differential dimension polynomials and a fundamental theorem on differential modules, Amer. J. Math. 91 (1969), 239-248.

[19] —, Kähler differentials and differential algebra, Ann. of Math. 89 (1969), 92-98.

[20] E. R. Kolchin, Differential Algebra and Algebraic Groups, Academic Press, New York, 1973.

[21] A. Krener, Normal forms for linear and nonlinear systems, Contemp. Math. 68 (1987), $157-189$. 
[22] S. Lang, Algebra, Addison-Wesley, Reading, 1971.

[23] P. Martin, Contribution à l'étude des systèmes différentiellement plats, Thèse, Ecole des Mines de Paris, 1992.

[24] H. Nijmeijer and A. J. van der Schaft, Nonlinear Dynamical Control Systems, Springer, New York, 1990.

[25] J. B. Pomet, C. H. Moog, and A. Aranda, A non-exact Brunovský form and dynamic feedback linearization, in: Proc. 31st CDC IEEE, Tucson, 1992, 2012-2017.

[26] J. F. Ritt, Differential Algebra, Amer. Math. Soc., New York, 1950.

[27] P. Rouchon, Necessary condition and genericity of dynamic feedback linearization, J. Math. Systems Estim. Control 4 (1994), 1-14.

[28] J. Rudolph, Une forme canonique en bouclage quasi statique, C. R. Acad. Sci. Paris Sér. I 316 (1993), 1323-1328.

[29] -, A canonical form under quasi-static feedback, in: Systems and Networks: Mathematical Theory and Applications, U. Helmke, R. Mennicken, and J. Saurer (eds.), 1993, 445-448.

[30] -, Viewing input-output system equivalence from differential algebra, J. Math. Systems Estim. Control 4 (1994), 353-383.

[31] _, Duality in time-varying linear systems: a module theoretic approach, Linear Algebra Appl., to appear.

[32] J. Rudolph and S. El Asmi, Filtrations and Hilbert polynomials in control theory, in: Systems and Networks: Mathematical Theory and Applications, U. Helmke, R. Mennicken, and J. Saurer (eds.), 1994, 449-452.

[33] W. Sluis, Absolute Equivalence and its Applications to Control Theory, PhD Thesis, University of Waterloo, 1992.

[34] M. Zeitz, Canonical forms for nonlinear systems, in: Nonlinear Control Systems Design, Selected Papers from the IFAC-Symposium, Capri/Italy 1989, A. Isidori (ed.), Pergamon Press, Oxford, 1990, 33-38. 